Culture of safety

\title{
Evaluating the culture of safety
}

\section{J Firth-Cozens}

\section{Leaders need to stay close to the action if their organisations are to be not just seen as safe, but actually to be safer}

W here once poor patient safety was deemed to be the result of individuals and technical inadequacy, ways of improving safety increasingly focus on the interaction of technology, human resources and organisations, together with the value systems or culture which lie behind them. ${ }^{1}$ In this issue of QSHC Pronovost et $a l^{2}$ describe the development of a scale from a tool which looked at cockpit management attitudes, with questions focusing very much on the leader's role in the enhancement of a safety culture. They found that staff saw their supervisors as having a greater commitment to safety than the more senior leaders.

Their emphasis on views of leadership-including management-is important. Leadership style in terms of personality and attitudes can have real consequences for safety ${ }^{3}{ }^{4}$ and for accurate reporting of error. ${ }^{5}$ It is not just personality or style that matters, however; the larger the gap between management's view of risks within their organisation and the views of the actual workers, the greater the number of actual accidents. ${ }^{6}$ Similarly, Pronovost et $a l^{2}$ show that management had a rather rosier view of safety in their hospital than did those most closely involved. Leaders need to stay close to the action if their organisations are to be not just seen as safe, but actually to be safer. Others have found that leadership influences employees' perceptions of the way safety is managed in their organisations, and those perceptions go on to influence their "on the job" behaviour and decisions which can lead to a lowering of actual accidents. ${ }^{7}$
These individual behaviours have been explored too in the operating theatre by Carthey et al. ${ }^{8}$ While they found that certain actions of individual surgeons-such as planning and adapting fast to change-affected outcomes, there were also a number of important organisational factors which influenced the process. For example, some organisations or teams within them had policies in place to reduce potential sources of distraction during surgery, to cut down non-case related communications to the surgeons, and to reduce the number of observers in theatre. Such policiesemanating from management-go on to form part of the culture of the theatre.

It is this everyday management commitment to safety which helps to make up a safety culture. This culture will also include wider organisational concepts such as the degree to which members report unsafe conditions, the speed of remedial action by management, the number of near miss reports, etcfactors which Cooper" called the "safety product", a useful dependent variable with which to assess any organisational improvements which occur after initiatives to increase safety.

However, the relatively sudden rise in the attention currently being given to patient safety should not mean that we focus on safety as a culture in itself, separate from quality and separate from the organisational culture as a whole. It exists within other aspects of the organisation and the wider culture, such as financial restraints, mergers, the skills market, political change, media attention, and so on. So staff instability in a theatre team has an effect on patient outcomes, ${ }^{8}$ and sleep loss affects cognitive skills and dexterity ${ }^{10}$ — both the result of wider organisational practices. In high risk organisations like health care, safety needs to be the dominant element of organisational culture rather than something which lies adjacent to but largely separate from the rest. ${ }^{9}$

Individuals can change their attitudes to safety, ${ }^{11}$ but this is unlikely to be maintained without the organisational commitment to safety being clearly strong. Pronovost et al ${ }^{2}$ show, however, that organisations can also change through feedback of survey results. A regular demonstration of the "products of safety" to staff and management alike may be a way to unite them in a single organisational goal to create the safest organisation possible.

Qual Saf Health Care 2003;12:401

\section{Author's affiliation}

J Firth-Cozens, The London Deanery, University of London, 33 Millman Street, London WCIN 3EJ, UK; jfirth-cozens@londondeanery.ac.uk

\section{REFERENCES}

1 Grote G, Küzler. Diagnosis of safety culture in safety management audits. Saf $\mathrm{Sc}$ 2000;34:131-50.

2 Pronovost PJ, Weast B, Holzmueller CG, et al. Evaluation of the culture of safety: survey of clinicians and managers in an academic medical center. Qual Saf Health Care 2003;12:405-10.

3 Firth-Cozens J, Mowbray D. Leadership and the quality of care. Qual Health Care 2001;10(Suppl II):ii3-7.

4 Chidester TR, Helmreick RL, et al. Pilot personality and crew coordination. Int J Aviat Psychol $1991 ; 1: 25-44$

5 Edmondson AC. Learning from mistakes is easier said than done: group and organisational influences on the detection and correction of human error. J Appl Behav Sci 1996;32:5-28.

6 Warren N. Worker-employer risk estimate disparity: identifying the unhealthy organisation. Work, Stress and Health '99: Organisation of Work in a Global Economy. Baltimore Conference, Maryland, 1999.

7 O'Toole M. The relationship between employees' perceptions of safety and organizational culture. J Saf Res 2002;33:231-43.

8 Carthey J, de Laval MR, Wright DJ, et al. Behavioural markers of surgical excellence. Saf Sci 2003;41:409-25

9 Cooper MD. Towards a model of safety culture. Saf Sci 2000;36:111-36.

10 Firth-Cozens J, Cording H. Do doctors' hours of work and sleep affect the quality of patient care? Qual Saf Health Care 2004 (in press).

11 Helmreich RL, Willhelm JA. Outcomes of crew resource management training. Int $J$ Aviat Psychol $1991 ; 1: 287-300$ 
Case record review

\section{Reviewing case record review}

\section{R McL Wilson}

\section{More public discussion is needed on the role and content of the case record}

ln their paper in this issue of QSHC in which they redesign current case (or medical) record review methods for the purpose of detecting adverse events and teasing out opportunities for preventing recurrence of this patient harm, Woloshynowych et al state that "in our view the full potential of retrospective record review has yet to be explored". Their view on the so far unrealised potential of the medical record is true, despite current efforts in some countries.

The medical record has a time honoured place as a chronological account of the clinical state and care of a particular patient. This place has been reinforced by the routine use of the medical record as part of any legal or insurance inquiry about care, as well as in clinical or peer review processes that are designed to improve the delivery of health care. But, unlike an incident report or computer alert, the medical record has a passive nature that requires and rewards systematic examination after an alerting process identifies the records that need review. That alert may be an adverse clinical outcome-for example, death of the patient-or of a procedural form such as a complaint or subpoena. For effective use of the medical record to improve health care, two elements are therefore needed-an alerting system to indicate records worthy of detailed examination and a systematic approach to that examination. The paper by Woloshynowych et al provides new insights into such a systematic approach to medical record review. It builds on the previous work that started with the Californian Insurance Feasibility Study ${ }^{2}$ to estimate the incidence of adverse events and adds questions from experience with clinical investigation of adverse events to better understand potential causes and contributory factors. This is a major step forward in using the medical record for improvement of health services, by understanding causation then generating recommendations for actions to prevent recurrence or alleviate effects of adverse events.
But what about the commonly cited shortcomings of the medical record? ${ }^{3}$ Availability and legibility of the record and reliability of the judgements made by reviewers are key areas for comment. The Quality in Australian Health Care Study ${ }^{4}$ reported that in only 24 out of 15000 records from 28 Australian hospitals was there insufficient information in the record to confirm a suspected adverse event, and that there was $80 \%$ agreement $(\chi=0.55)$ between two senior medical reviewers as to the presence or absence of an adverse event in the remaining records. From similar work in New Zealand, the reliability of determination of adverse events was $87.5 \%$ agreement $(\chi=0.47) .^{5}$ These findings indicate that case record review performs better than any other currently available modality for detecting the frequency of adverse events to patients. But it is also clear that performance could be better.

Given the fundamental importance of successful case record review and its progression towards automation with the electronic health record, the patchy success of case record policy is both surprising and disappointing. When this is combined with relative lack of investment in the content and accessibility of these records, it is not surprising that records do not enjoy the reputation that this unique data source should have. The following list of essential elements comes from the reviewers in the Quality in Australian Health Care Study ${ }^{6}$ :

- Medical admission.

- Medical discharge.

- Discharge summary.

- Medical continuation notes.

- Management or treatment plans.

- Consultations.

- Results of investigations.

- Short stay documentation.

- Referral/follow up letters from GPs or specialists.

- All volumes of the medical record simultaneously accessible.

They further recommended:
- Standardise the layout of the medical record

- Use admission dividers as a standard requirement for medical record filing.

- Devise a policy for loose sheet filing.

- Use an integrated medical record for all healthcare professionals.

- Keep all records relating to a patient in a single medical record rather than divided between clinics or sites of care.

- Devise standardised systems for linking mother and baby records after discharge.

If we could add the suggestions from Woloshynowych et al on detecting and preventing adverse events, then we could further improve the quality and usefulness of medical records. Even allowing for the fact that these latter recommendations need further formal evaluation and probable refinement, what is really lacking is the leadership to improve the usefulness of medical records for improvement. The incentives for many of the contributors to the medical record to improve their contribution consistent with particular standards are few and the barriers may seem large. If we then automate a current "faulty" system of medical records into an "electronic health record" we can only expensively perpetuate the problem.

We are now at a crucial time where more public discussion on the role and content of the medical record is required, and it is not clear where the leadership for this work is located. Once agreed, then appropriate standards can be internationally agreed and local incentives for implementation can be elucidated. There are current standards in this area, but it is not clear whether they take into account all the necessary functions of the record, especially the role of the record in quality improvement. Even when standards are in place-for example, through accreditation in the US or Australia-it is not obvious that these standards are being followed. Perhaps the readers of this journal could help this discussion gain momentum by using the rapid response function http://qhc.bmjjournals.com/cgi/ eletter-submit/12/6/402 to answer three questions:

1. What are the key functions of the medical record?

2. What are the key elements that would need to be included in a medical record in order to achieve these functions?

3. How do we implement the recommendations from 2 above? 
You, as the readers of $Q S H C$, can make a difference here.

\section{Qual Saf Health Care 2003;12:402-403}

Correspondence to: R McL Wilson, Chairman, NSW Council for Quality in Health Care, Royal North Shore Hospital, NSW 2065, Australia; rwilson@doh.health.nsw.qov.au

\section{REFERENCES}

1 Woloshynowych M, Neale G, Vincent C. Case record review of adverse events: a new approach. Qual Saf Health Care 2003;12:41 1-5.

2 Mills DH. Medical insurance feasibility study. A technical study. West J Med 1978;128:360-5

3 Neale G, Woloshynowych M. Retrospective case record review: a blunt instrument that needs sharpening. Qual Saf Health Care 2003;12:2-3.
4 Wilson RM, Runciman WR, Gibberd RW, et al The Quality in Australian Health Care Study. Med J Aust 1995;163:458-71.

5 Davis $\mathbf{P}$, Lay-Yee R, Briant R, et al. Preventable inhospital medical injury under the "no-fault" system in New Zealand. Qual Saf Health Care 2003; 12:251-6

6 Quality in Australian Health Care Study (QAHCS). Report from QAHCS Consortium to the Commonwealth Department of Health and Human Services. 1996; Volume 2.
It may therefore be that, despite the impressive array of mechanisms in place, a focus on governance arrangements in terms of policy implementation is misplaced. Instead, the focus should be shifted much further down to the level where patients interact with healthcare processionals. As Coulter points out: "Instead of beginning at the rarefied level of formal involvement in organisations and official committees, implementation of the policy to promote patient centred care should have started much nearer the ground at the point where patients and professionals interact". " It is therefore of crucial importance to develop and disseminate effective methodology which will pick up and act on patients' perceptions of the quality of such encounters, such as that described in the paper by Entwistle and colleagues in this issue of QSHC. Their paper will certainly be of interest to all those working to develop similar methodology in other healthcare systems internationally. There is no doubt that the "new NHS" is struggling to be user responsive; only by thoughtful improvements to the methodology and approach used in primary care will it be possible to rectify the situation.

\section{Qual Saf Health Care 2003;12:403-404}

Correspondence to: S Pickard, National Primary Care Research and Development Centre (NPCRDC), University of Manchester, Manchester M16 8GB, UK; susan.pickard@man.ac.uk ment means that PCTs often express uncertainty as to how to do it (despite the excellent guidance produced by the NHS Consumers in Research Support Unit). ${ }^{7}$ The role of non-executive directors on the board and their relationship to other mechanisms for channelling user involvement is characterised by a lack of clarity, with a similar ambiguity in practice concerning the place of the board in relation to the Professional Executive Committee (PEC). At the same time it is ironical that, despite the abundant monitoring processes set up to evaluate the performance of various healthcare providers, this sort of information may not be telling the user anything he or she would actually wish to know (such as whether a GP has good interpersonal skills or what the patient recognises to be a "good doctor"). ${ }^{8}$ 
7 Consumers in NHS Research Support Unit. Involving consumers in research and development in the NHS: briefing notes for researchers. Winchester: Help for Health Trust 2000.
8 Strathern M, ed. Audit cultures. London: Routledge, 2000.

9 Coulter A. Whatever happened to shared decision-making? Health Expectations 2002;5:185-6.
10 Entwhistle VA, Andrew JE, Conniff AO. Public opinions about systems for "feeding back" views to the National Health Service. Qual Saf Health Care 2003;12:435-42.

Error classification

\section{Classifying and identifying errors}

\section{A J Avery}

\section{There are no easy answers to developing an all encompassing medical error classification system in primary care}

C lassifications can help us to make sense of the world and, in the field of medical errors, they can help us to assess what are the most important problems. There have been numerous attempts to develop classifications of medical error, although relatively few of these have come from primary care. This commentary highlights some issues in the classification of medical errors and considers the case for more systematic attempts to determine the incidence of these errors.

One of the problems with classifying medical errors is that there are so many ways of doing it. For example, one can focus on processes such diagnosis ${ }^{1}$ or describe underlying system failures. ${ }^{2}$ Also, one can classify errors in terms of the types of disease, drug, or procedure most commonly associated with error or in terms of the severity of outcomes.

The paper by Rubin and colleagues ${ }^{3}$ in this issue of QSHC provides a classification containing six categories of error. The classification was based on broad themes coming out of the analysis of 65 events and it may prove to be useful in primary care along with other classifications. ${ }^{4}$ In common with other classification systems, there is room for overlap between categories. For example, a "clinical error" might also be a "communication error". Some investigators have tried to get around this problem by "cross cutting" between different types of classification. For example, Bhasale and colleagues ${ }^{1}$ have classified errors in primary care in terms of types of incident such as those relating to prescribing or diagnosis. They have then described contributing factors such as communication problems or errors of judgement. Nevertheless, Bhasale et al note that there is still overlap between some of the categories that they developed.
Most attempts to classify errors in primary care have been based upon the reporting of incidents by health professionals. ${ }^{13-5}$ This type of approach provides valuable information but it is problematic because of under reporting and reporting bias. This means that classifications will be based on incomplete data and estimates of the incidence of error are likely to be inaccurate. It is therefore worth asking if there are better alternatives.

There is little doubt that the reporting of incidents by health professionals can provide useful feedback on errors. This is the approach that is being adopted by the National Patient Safety Agency in the UK with the aim of helping the NHS to learn more from mistakes. This type of approach has been used successfully in other industries and there are good reasons to expect it to have a positive impact in health care. Nevertheless, a more systematic approach to identifying errors could provide better evidence on which to identify priorities for action. This has occurred in the field of prescribing in primary care $^{5}$ where, for example, a recent prospective cohort study has examined the frequency of preventable adverse events resulting from prescriptions, ${ }^{6}$ and another study has identified the errors in medicines management associated with preventable medication related admissions to hospital. ${ }^{7}$

In secondary care there have been a number of descriptive studies documenting the nature and scale of errors. Rather than using incident reports from healthcare professionals, many of these studies have worked on the basis of detailed review of patients' records, sometimes with further information being collected from healthcare staff. Review of patients' records could be used in primary care, although there might be problems due to incomplete documentation. A combination of record review and interviews with staff and patients might therefore be more successful in providing the information needed to judge whether errors have occurred and their underlying causes. This approach would lend itself particularly well to analysis of serious adverse events such as hospital admissions, but it might also work if a study were to be done of all healthcare encounters in a primary care setting. Even so, this approach would not pick up errors such as the equipment failures identified by Rubin and colleagues. ${ }^{3}$

It seems, therefore, that there are no easy answers when it comes to the development of all encompassing classification systems for medical error in primary care. Approaches based on selfreporting clearly provide useful information and more robust descriptive studies would still not give the full picture. Nevertheless, I think it would be worthwhile undertaking more systematic analyses of primary care encounters to determine the most important errors and how frequently they occur.

Qual Saf Health Care 2003;12:404

\section{Author's affiliation}

A J Avery, School of Community Health Sciences, The Medical School, Queen's Medical Centre, Nottingham NG7 2UH, UK; tony.avery@nottingham.ac.uk

\section{REFERENCES}

1 Bhasale AL, Miller GC, Reid SE, et al. Analysing potential harm in Australian general practice: an incident-monitoring study. Med J Aust 1998;169:73-6.

2 Leape LL, Bates D, Cullen DJ, et al. Systems analysis of adverse drug events. JAMA 1995;274:35-43.

3 Rubin G, George A, Chinn DJ, et al. Errors in general practice: development of an error classification and pilot study of a method for detecting errors. Qual Saf Health Care 2003;12:443-7.

4 Dovey SM, Meyers DS, Phillips RL, et al A preliminary taxonomy of medical errors in family practice. Qual Saf Health Care 2002;1 1:233-8.

5 Sanders J, Esmail A. The frequency and nature of medical error in primary care: understanding the diversity across studies. Fam Pract 2003;20:231-6

6 Gandhi TK, Weingart SN, Borus J, et al. Adverse drug events in ambulatory care. $N$ Engl J Med 2003:348: 1556-64

7 Howard RL, Avery AJ, Howard PD, et al Investigation into the reasons for preventable drug related admissions to a medical admissions unit: observational study. Qual Saf Health Care 2003;12:280-5. 